IMAGE IN

CARDIOLOGY

\title{
Critical thinking in cardiovascular imaging: hypertrophic cardiomyopathy and anterior mitral valve cleft
}

\section{Thadathilankal-Jess John*, W.M.J. Bodenstein" and} Philip Herbst*

\section{"Division of Cardiology, Stellenbosch University and Tygerberg Academic Hospital, Bellville, South Africa \\ "Panorama Mediclinc, Panorama, Cape Town, South Africa}

Address for correspondence:

DrThadathilankal-Jess John

Division of Cardiology

Department of Medicine

Stellenbosch University and Tygerberg Academic Hospital

PO Box 24I

Cape Town

8000

South Africa

Email:

jessjohnt@gmail.com

A 72-year-old female with a longstanding history of dyspnoea was found to have left ventricular hypertrophy $(\mathrm{LVH})$ on echocardiography out of proportion to the severity of her well-controlled hypertension. In addition, there was evidence of an intraventricular gradient with mitral regurgitation (MR) which could not be further characterised due to poor imaging windows. She was referred for a cardiac MRI (CMR) to elucidate the cause of her $L V H$ as well as assess the severity of her MR. CMR confirmed hypertrophic cardiomyopathy (HCM) with marked asymmetric septal hypertrophy (maximal septal diameter $18 \mathrm{~mm}$ ) and systolic anterior motion (SAM) of the mitral valve (MV) with associated severe left ventricular outflow obstruction (Figure I). Additionally, LV volumes were small and ejection fraction was supranormal (80\%). Phase contrast imaging confirmed moderate MR with regurgitant volume and fraction measuring $28 \mathrm{ml} /$ beat and $28 \%$ respectively. The MR mechanism appeared to be related to mitral valve SAM (Video I). However, the MR jet direction was centrally directed whereas SAM related MR is typically posteriorly directed.(1) Further evaluation of our patient's MV revealed a small cleft in the anterior mitral valve leaflet (AMVL) at the A2 - A3 transition (Figure 2, Video 2). This was associated with leaflet tip thickening and mild calcification. This case demonstrates the utility of CMR in assessing both morphological and functional

\section{ABSTRACT}

We present a case of a 72-year-old female with hypertrophic cardiomyopathy with systolic anterior motion (SAM) of the mitral valve and secondary mitral regurgitation (MR). The jet direction was noted to be centrally directed which is atypical for SAM associated MR. A search for a secondary cause revealed a mitral valve cleft at the A2/3 transition zone. This case highlights the importance of critical thinking in cardiovascular imaging. SAHeart 2021;|8:150-15|

deficits in patients with HCM. This accurate delineation of pathology, especially underlying mitral valve pathology in HCM is important as these patients may require concurrent mitral valve repair at the time of septal myectomy in patients with ongoing symptoms despite medical therapy. It further highlights the importance of assessing the jet direction in patients with SAM related MR as it is expected to be posteriorly directed. A thorough search for additional mechanisms should be carried out when jet direction is found to be anteriorly or centrally directed.

\section{FUNDING}

No external funding was received for preparation of this manuscript.

\section{AUTHOR INVOLVEMENT}

All involved authors played a role in writing the manuscript.

Conflict of interest: none declared.

\section{REFERENCES}

I. Hang D, Schaff HV, Nishimura RA, et al. Accuracy of jet direction on doppler echocardiography in identifying the etiology of mitral regurgitation in obstructive hypertrophic cardiomyopathy. J Am Soc Echocardiogr. 2019 ;32(3):333-40. 


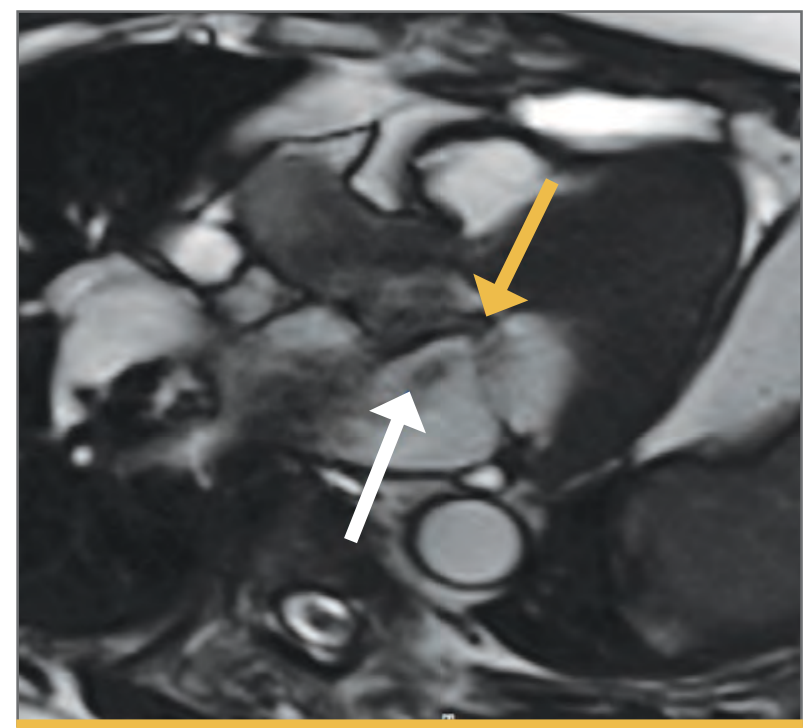

FIGURE I: Apical 3-chamber showing systolic anterior motion of the mitral valve (orange arrow) with severe left ventricular outflow tract obstruction and a centrally directed mitral regurgitant jet (white arrow).

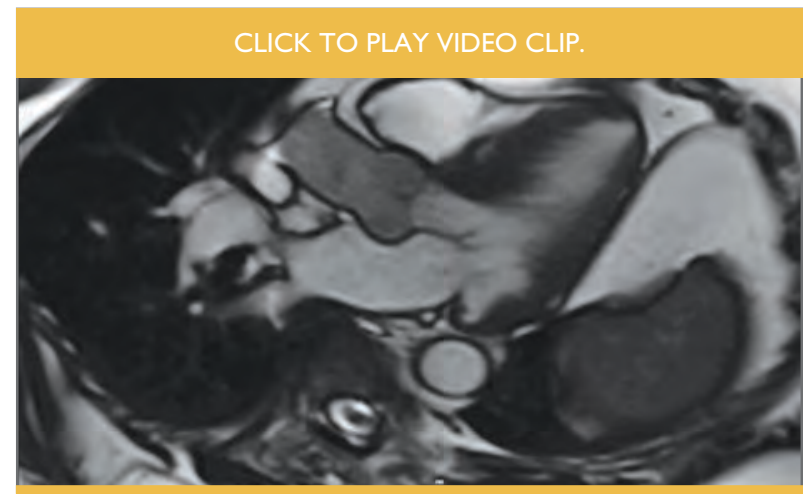

VIDEO I: Three chamber cine loop depicting evidence of SAM and outflow tract obstruction with an centrally directed mitral regurgitation jet.

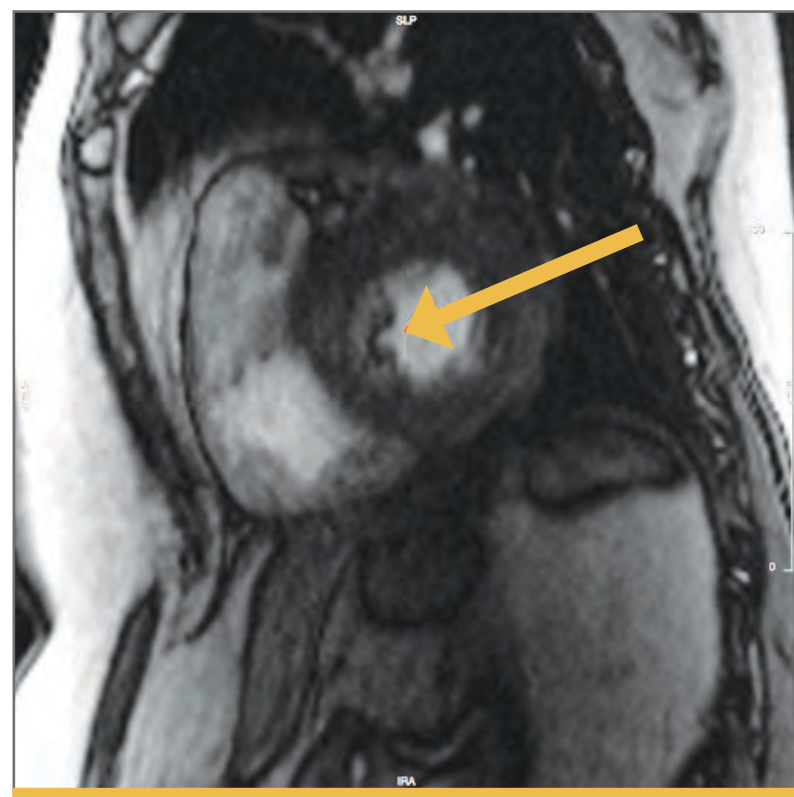

FIGURE 2: Mitral valve short-axis images revealing anterior mitral valve cleft at the A2/A3 transition zone.

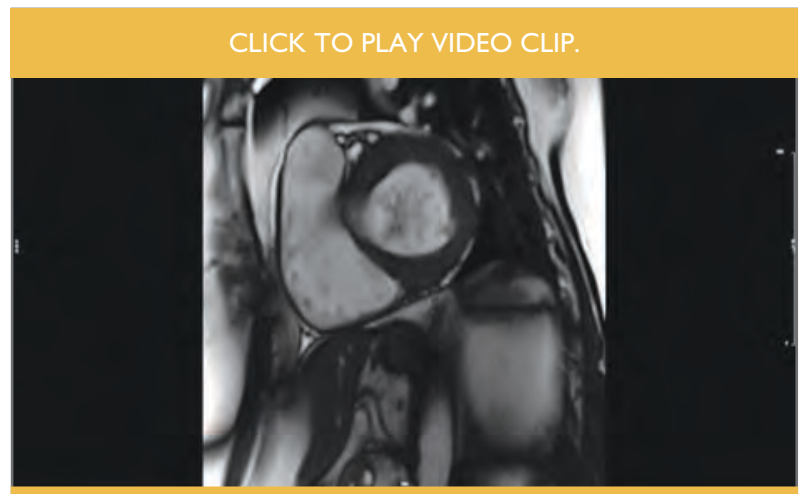

VIDEO 2: Short-axis cine loop through the mitral valve demonstrating an anterior mitral valve cleft at the A2/3 transition zone. 